\title{
Complex-mediated microwave-assisted synthesis of polyacrylonitrile nanoparticles
}

This article was published in the following Dove Press journal:

Nanotechnology, Science and Applications

19 October 2010

Number of times this article has been viewed

\section{Trinath Biswal \\ Ramakanta Samal \\ Prafulla K Sahoo}

Department of Chemistry, Utkal University, Vani Vihar, Bhubaneswar 751004 , India
Correspondence: Prafulla K Sahoo

Department of Chemistry,

Utkal University, Vani Vihar,

Bhubaneswar 751004, India

$\mathrm{Fax}+916742585018$

Email psahoochemuu@satyam.net.in
Abstract: The polymerization of acrylonitrile (AN) is efficiently, easily, and quickly achieved in the presence of trans-[Co(III)en $\left.{ }_{2} \mathrm{Cl}_{2}\right] \mathrm{Cl}$ complex in a domestic microwave (MW) oven. MW irradiation notably promoted the polymerization reaction; this phenomenon is ascribed to the acceleration of the initiator, ammonium persulfate (APS), decomposition by microwave irradiation in the presence of $\left[\mathrm{Co}(\mathrm{III}) \mathrm{en}_{2} \mathrm{Cl}_{2}\right] \mathrm{Cl}$. The conversion of monomer to the polymer was mostly excellent in gram scale. Irradiation at low power and time produced more homogeneous polymers with high molecular weight and low polydispersity when compared with the polymer formed by a conventional heating method. The interaction of reacting components was monitored by UV-visible spectrometer. The average molecular weight was derived by gel permeation chromatography (GPC), viscosity methods, and sound velocity by ultrasonic interferometer. The uniform and reduced molecular size was characterized by transmission electron microscopy, the diameter of polyacrylonitrile nanoparticles (PAN) being in the range 50-115 nm and 40-230 $\mathrm{nm}$ in microwave and conventional heating methods respectively. The surface morphology of PAN prepared by MW irradiation was characterized by scanning electron microscope (SEM). From the kinetic results, the rate of polymerization $\left(\mathrm{R}_{\mathrm{p}}\right)$ was expressed as $\mathrm{R}_{\mathrm{p}}=[\mathrm{AN}]^{0.63}[\mathrm{APS}]^{0.57}$ [complex (I)]..$^{0.88}$

Keywords: microwave, complex catalyst, nanoparticle, kinetics

\section{Introduction}

Due to increasing environmental concern, green chemistry has been receiving progressively more attention since $1990 .{ }^{1,2}$ Therefore, there is a great necessity for the advancement of novel methodologies for chemical reactions using an environmentally eco-friendly method. A promising approach from this standpoint is to search for new processes and technologies which, from the very beginning, are aimed at sharply curtailing environmental pollution by reducing the volumes of chemical production wastes.

Microwave (MW) irradiation has been applied to various chemical reactions. Many review papers have been published about MW-assisted chemical reactions., ${ }^{3,4}$ The MW method provides a fast and direct heating method. In many cases, it can dramatically reduce reaction times from hours to minutes, increase product yields, and enhance product purity. ${ }^{5}$

One major purpose of this study is to prepare polyacrylonitrile nanoparticles (PAN) by novel, low cost, and effective $\left[\mathrm{Co}(\mathrm{III}) \mathrm{en}_{2} \mathrm{Cl}_{2}\right] \mathrm{Cl}$ complex catalysis under microwave irradiation, as no reports are available to date. Also, reports on the kinetics of the MW polymerization initiated by ammonium persulfate (APS) in the presence of the transition metal complex are limited. The complex has a pronounced effect 
in decreasing the particle size to the nano-range, besides stabilizing the polymer latex leading to a high conversion. The current system is of great interest as it is found to result in the formation of particles of nano-range and nano-technology reaching its peak in the current era.

\section{Materials and methods Materials}

Acrylonitrile (AN) monomer (E. Merck, Mumbai, India) was purified as reported earlier. ${ }^{6}$ The initiator (APS) and all other reagents like Cobalt (II) Chloride, ethylenediamine (en), etc. (E. Merck) were all analytical reagent (AR) grade and were used after purification by standard techniques.

\section{Preparation of $\left[\mathrm{Co}(\mathrm{III}) \mathrm{en}_{2} \mathrm{Cl}_{2}\right] \mathrm{Cl}$ complex}

The $\left[\mathrm{Co}(\mathrm{III}) \mathrm{en}_{2} \mathrm{Cl}_{2}\right] \mathrm{Cl}$ complex was prepared by treating $\mathrm{Co}$ (II) $\mathrm{Cl}_{2} 6 \mathrm{H}_{2} \mathrm{O}$ with $10 \%$ solution of en using the method available in literature. ${ }^{7}$ A solution of en in water was slowly added in, with constant stirring to a solution of $\mathrm{Co}(\mathrm{II}) \mathrm{Cl}_{2}$ $6 \mathrm{H}_{2} \mathrm{O}$ in water. Cobalt was oxidized by bubbling a vigorous stream of air through the solution for 9-10 hours and then concentrated hydrochloric acid (conc. HCL) was then added to the oxidized solution. The solution was allowed to evaporate on a steam bath until a crust began to form over the surface. This was then cooled and allowed to stand overnight, when bright green platelets of the hydrochloride of the complex were separated out. The crystals were filtered off, washed with ether, recrystallized in the presence of conc. $\mathrm{HCl}$ and then dried at $100^{\circ} \mathrm{C}$ until a solution of the complex in distilled water was neutral to litmus. The formation of the complex trans-[Co(III)en $\left.{ }_{2} \mathrm{Cl}_{2}\right] \mathrm{Cl}$ was confirmed by UV-visible spectral analysis (Scheme 1).

\section{Synthesis of PAN nanoparticles By microwave oven}

The polymerization experiments were carried out in a Kenstar MOW 9811, 1200 W domestic MW oven (Kenstar, Maharashtra, India) which was used for all the experiments. The average bulk temperature at end of the reaction was measured by inserting a thermometer into the reaction mixture. All the experiments were done with water and benzene as solvent and the temperature at less than $100^{\circ} \mathrm{C}$. For all the MW powers studies, the exposure time was varied from 30 to $180 \mathrm{sec}$. The reactions were carried out with the requisite amount of $\left[\mathrm{Co}(\mathrm{III}) \mathrm{en}_{2} \mathrm{Cl}_{2}\right] \mathrm{Cl}$ monomer $\mathrm{AN}$ and APS in $\mathrm{N}_{2}$ atmosphere. The particle size distribution parameters are derived from the number-average particle diameter $(D n)$ shown as a preliminary study in Table 2 .

\section{By conventional heating}

The polymerization was carried out in a reaction vessel containing known concentrations of $\left[\mathrm{Co}(\mathrm{III}) \mathrm{en}_{2} \mathrm{Cl}_{2}\right] \mathrm{Cl}$ and the monomer $\mathrm{AN}$ in $\mathrm{N}_{2}$ atmosphere. The solutions were stirred at 400-500 rpm, which helped in the formation of micelles in the complex medium. After maintaining a constant temperature of $50^{\circ} \mathrm{C}$, the requisite amount of initiator solution was carefully injected to the reaction mixture.

After the desired time, both in microwave oven and conventional heating, the polymerization was terminated by keeping the reaction vessel in ice-cold water and by adding a known excess of hydroquinone, which spontaneously consumed the unreacted free radicals. ${ }^{8}$ The precipitated polymers were filtered and purified by washing repeatedly with distilled water and absolute alcohol. These were then dried at $40^{\circ} \mathrm{C}$ until a constant weight. The percentage conversion and the rate of polymerization $\left(R_{p}\right)$ were determined gravimetrically.

\section{Characterization}

The visible spectra of $\left[\mathrm{Co}(\mathrm{III}) \mathrm{en}_{2} \mathrm{Cl}_{2}\right] \mathrm{Cl}$ complex vis-à-vis those of the monomer and the initiator were studied using a Perkin Elmer Lambda-20 UV-visible spectrophotometer (Perkin Elmer, Waltham, MA). The average molecular weights $\left(\bar{M}_{n}\right)$ of the purified samples were determined by widely used methods such as gel permeation chromatography (GPC) and intrinsic viscosity $\left(\bar{M}_{v}\right)$ using the relationship of Mark-Houwink-Sakkurada ${ }^{9}$ in pure benzene at $30^{\circ} \mathrm{C}$ where $[\eta]=5.20 \times 10^{-5} \mathrm{M}_{v}^{0.7}$. Sound velocity of the samples in solution was measured with an ultrasonic interferometer (Mittal Enterprises, New Delhi, India). Nano-scale structure of PAN was investigated by means of a H-7100 transmission electron microscope (TEM) (Hitachi High-Tech, Tokyo, Japan), operated at an accelerating voltage of $100 \mathrm{Kv}$. The surface morphology of the samples was observed with a JEOL 5200 scanning electron microscope (SEM) (JEOL, Tokyo, Japan) after coating it with gold at different magnifications.

\section{Results and discussion}

The results of the study on the polymerization of AN initiated by APS catalyzed by $\left[\mathrm{Co}(\mathrm{III}) \mathrm{en}_{2} \mathrm{Cl}_{2}\right] \mathrm{Cl}$ are presented in Table 1. From the results, it is evident that $\left[\mathrm{Co}(\mathrm{III}) \mathrm{en}_{2} \mathrm{Cl}_{2}\right] \mathrm{Cl}$ gave the maximum conversion $\%$ and highest $\mathrm{R}_{\mathrm{p}}$. The order of $\mathrm{R}_{\mathrm{p}}$ for the various systems is as follows:

$$
\mathrm{R}_{\mathrm{p}}\left(\mathrm{APS}+\left[\mathrm{Co}(\mathrm{III}) \mathrm{en}_{2} \mathrm{Cl}_{2}\right] \mathrm{Cl}\right)>\mathrm{R}_{\mathrm{p}}(\mathrm{APS})
$$


a

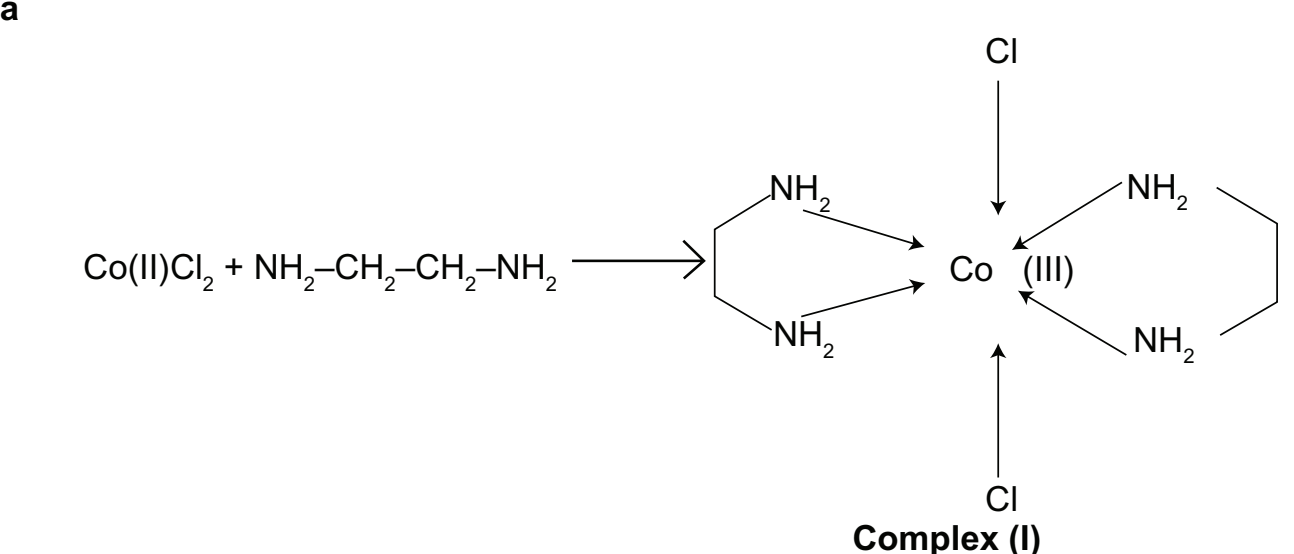

b

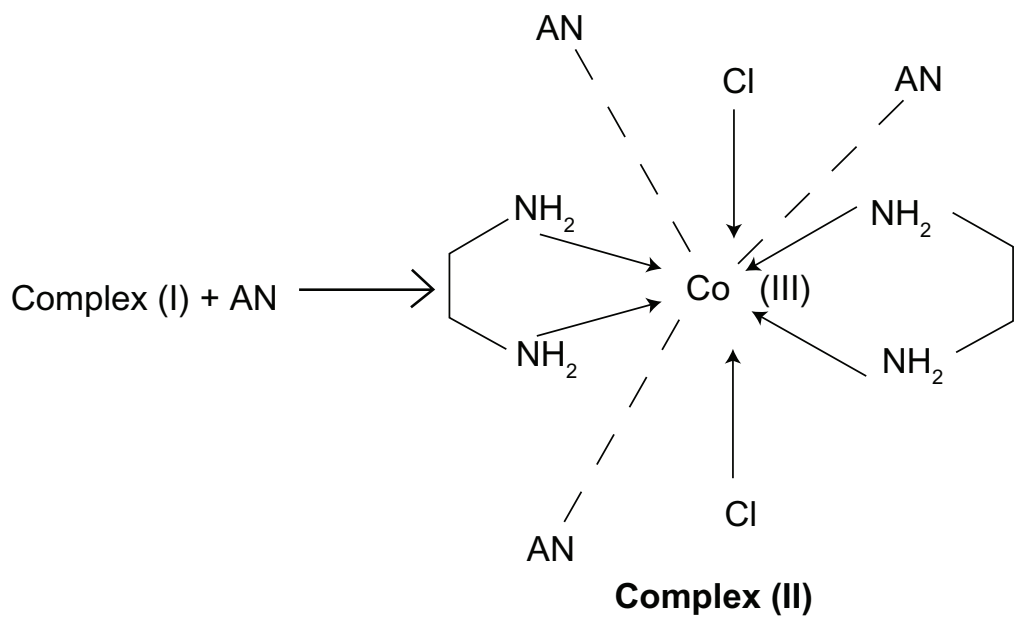

Scheme I a) Formation of $\left[\mathrm{Co}(\mathrm{III}) \mathrm{en}_{2} \mathrm{Cl}_{2}\right] \mathrm{Cl}$ complex (I), and b) coordination of monomer acrylonitrile (AN) with $\left[\mathrm{Co}(\mathrm{III}) \mathrm{en}_{2} \mathrm{Cl}_{2}\right] \mathrm{Cl}$.

\section{UV-visible spectra}

The UV-visible spectra of various mixtures like $\mathrm{CoCl}_{2}$, $\left[\mathrm{Co}(\mathrm{III}) \mathrm{en}_{2} \mathrm{Cl}_{2}\right] \mathrm{Cl}$, [Co(III)en $\left.{ }_{2} \mathrm{Cl}_{2}\right] \mathrm{Cl} / \mathrm{APS}$ and $\left[\mathrm{Co}(\mathrm{III}) \mathrm{en}_{2} \mathrm{Cl}_{2}\right]$ $\mathrm{Cl} / \mathrm{AN} / \mathrm{APS}$, before and after reaction by $\mathrm{MW}$, were carried out in an aqueous solution to obtain a complete picture of the interaction between the reacting species and their relationship with the rate data (Figure 1). The bidentate ligand character of en with colored cations like $\mathrm{Co}$ (III) to form chelate complex is well recognized, ${ }^{10}$ where the $\mathrm{Co}(\mathrm{III})$ and en ratio in complex Scheme 1a is 1:2, as evidenced by the proportionality found in the rate expression (Figure 1b). The high conversion may be ascribed to the high rate of production of initiating radicals by facile homolysis of the initiator bound by transient complex trans- $\left[\mathrm{Co}(\mathrm{III}) \mathrm{en}_{2} \mathrm{Cl}_{2}\right] \mathrm{Cl}$. Further, the central metal ion, $\mathrm{Co}$ (III), is coordinated to three molecules of monomer (AN), (Complex-II, Scheme 1b). With the addition of the initiator (APS) to the above mixture (Complex-II), the absorbance reduces from 1.32 (Figure 1b) to 1.06 (Figure 1c), showing the initiation of polymerization, thus establishing the mechanism of complex initiation as evidenced by the change in absorbance from 1.06 (Figure 1c) to 0.75 (Figure 1d) after polymerization. The high conversion values with $\left[\mathrm{Co}(\mathrm{III}) \mathrm{en}_{2} \mathrm{Cl}_{2}\right] \mathrm{Cl}$ complex may be due to the high rate of production of initiating radicals generated by the heterolysis of the initiator, bound by a complex of [Co(III) $\left.\mathrm{en}_{2} \mathrm{Cl}_{2}\right] \mathrm{Cl}$, where the internal energy is transferred to the monomer. The new, non-conventional complex-initiated system leads to stabilizing of the emulsion latex to a high conversion in the absence of an added emulsifier.

It was found that the conversion and the $\mathrm{R}_{\mathrm{p}}$ were strongly affected by the monomer, initiator and complex concentrations and also by the reaction time. The detailed kinetics are discussed below.

\section{Variation of time}

Figure 2 shows the variation and comparison of conversion with reaction time under microwave and traditional heating process, keeping the concentrations of AN, APS, and [Co(III) 
Table I Effect of concentration of AN, APS, and $\left[\mathrm{Co}(\mathrm{III}) \mathrm{en}_{2} \mathrm{Cl}_{2}\right] \mathrm{Cl}$ on the \% conversion and $\mathrm{Rp}$ by microwave and conventional processes and on Mv and $\mathrm{Mn}$ by viscosity and GPC methods of PAN

\begin{tabular}{|c|c|c|c|c|c|c|c|c|}
\hline \multirow{2}{*}{$\frac{[\mathrm{AN}] \times 10^{-2}}{\mathrm{~mol} \mathrm{dm}}$} & \multicolumn{2}{|c|}{$\left[\mathrm{Co}(\mathrm{III}) \mathrm{en}_{2} \mathrm{Cl}_{2}\right] \mathrm{Cl}$} & \multicolumn{2}{|c|}{ MW irradiation process } & \multicolumn{2}{|l|}{ Heating process } & \multicolumn{2}{|c|}{$\begin{array}{l}\text { Molecular weight } \\
\text { by MW process }\end{array}$} \\
\hline & $\mathrm{mol} \mathrm{dm}^{-3}$ & $\times 10^{-3} \mathrm{~mol} \mathrm{dm}^{-3}$ & Conversion (\%) & $R_{p} \times 10^{6} \mathrm{dm}^{-3} \mathrm{~s}^{-1}$ & Conversion (\%) & $R_{p} \times 10^{8} \mathrm{dm}^{-3} \mathrm{~s}^{-1}$ & $M_{v} \times 10^{-6}$ & $M_{n} \times 10^{-6}$ \\
\hline 0 & 10.0 & 10.0 & 0.0 & 0.0 & 0.0 & 0.0 & - & - \\
\hline 0.5 & 10.0 & 10.0 & 86.22 & 3.6 & 62.89 & 6.90 & 1.07 & 0.87 \\
\hline 1.0 & 10.0 & 10.0 & 87.16 & 7.45 & 67.32 & 12.43 & 1.45 & 1.0 \\
\hline 1.5 & 10.0 & 10.0 & 88.12 & 9.98 & 70.43 & 15.45 & 1.78 & 1.22 \\
\hline 2.0 & 10.0 & 10.0 & 90.46 & 10.92 & 71.90 & 16.56 & 2.03 & 1.54 \\
\hline 2.5 & 10.0 & 10.0 & 89.0 & 11.45 & 70.34 & 17.56 & 2.34 & 1.78 \\
\hline 3.0 & 10.0 & 10.0 & 87.43 & 12.32 & 69.98 & 18.87 & 2.65 & 2.01 \\
\hline 3.5 & 10.0 & 10.0 & 84.56 & 12.89 & 67.67 & 19.67 & 2.89 & 2.34 \\
\hline 2.0 & 0 & 10.0 & 0.0 & 0.0 & 0.0 & 0.0 & & \\
\hline 2.0 & 2.5 & 10.0 & 67.84 & 5.67 & 56.65 & 14.78 & & \\
\hline 2.0 & 5.0 & 10.0 & 75.98 & 8.5 & 65.89 & 15.89 & & \\
\hline 2.0 & 10.0 & 10.0 & 90.46 & 10.92 & 71.90 & 16.56 & & \\
\hline 2.0 & 15.0 & 10.0 & 91.02 & 11.56 & 72.21 & 16.97 & & \\
\hline 2.0 & 20.0 & 10.0 & 91.34 & II.87 & 72.87 & 17.22 & & \\
\hline 2.0 & 25.0 & 10.0 & 91.78 & 12.02 & 73.03 & 17.99 & & \\
\hline 2.0 & 10.0 & 0 & 5.98 & 0.12 & 1.23 & 0.20 & & \\
\hline 2.0 & 10.0 & 2.5 & 69.98 & 5.67 & 49.98 & 9.12 & & \\
\hline 2.0 & 10.0 & 5.0 & 80.45 & 8.23 & 51.23 & 11.34 & & \\
\hline 2.0 & 10.0 & 10.0 & 90.46 & 10.92 & 68.85 & 14.32 & & \\
\hline 2.0 & 10.0 & 15.0 & 90.98 & 11.89 & 71.90 & 16.56 & & \\
\hline 2.0 & 10.0 & 20.0 & 91.34 & 12.43 & 72.23 & 18.20 & & \\
\hline 2.0 & 10.0 & 25.0 & 91.56 & 12.86 & 72.67 & 18.65 & & \\
\hline
\end{tabular}

Notes: Polymerization rates (Rp) are presented for microwave oven for $240 \mathrm{~s}$ and for conventional heating at $50^{\circ} \mathrm{C}$ for $3 \mathrm{~h}$.

Abbreviations: AN, acrylonitrile; APS, ammonium persulfate; Rp, rate of polymerization; Mv, viscosity average molecular weight; Mn, number average molecular weight; GPC, gel permeation chromatography; PAN, polyacrylonitrile nanoparticles; MW, microwave.

en $\left.\mathrm{Cl}_{2}\right] \mathrm{Cl}$ constant at $2 \times 10^{-2}, 10 \times 10^{-3}$, and $10 \times 10^{-3} \mathrm{~mol} \mathrm{dm}^{-3}$, respectively. It was found that the polymerization reaction is very fast in the microwave oven and shows a dead end polymerization tendency, ie, the initiation activity of the initiator is high in the initial period and hence, after $240 \mathrm{~s}$, it increases at a comparatively slow rate. But in the case of traditional heating, more time is required, ie, $3 \mathrm{~h}$ for production as compared to microwave irradiation.

Table 2 Variation of particle size in microwave and conventional processes at different concentrations of complex at [AN] = $2.0 \times 10^{-2} \mathrm{~mol} \mathrm{dm}^{-3},[$ APS $]=10.0 \times 10^{-3} \mathrm{~mol} \mathrm{dm}^{-3}$

\begin{tabular}{lll}
\hline$\left[\mathrm{Co}(\mathrm{III}) \mathrm{en}_{2} \mathrm{Cl}_{2}\right] \mathrm{Cl}$ & \multicolumn{2}{l}{ Diameter of particle $(\mathrm{nm})$} \\
\cline { 2 - 3 }$\left(\mathrm{mol} \mathrm{dm}^{-3}\right)$ & $\begin{array}{l}\text { Microwave } \\
\text { process }\end{array}$ & $\begin{array}{l}\text { Conventional } \\
\text { process }\end{array}$ \\
\hline 0 & 347 & 454 \\
2 & 50 & 40 \\
4 & 67 & 86 \\
8 & 79 & 98 \\
12 & 88 & 111 \\
16 & $10 \mathrm{I}$ & 122 \\
20 & 115 & 147 \\
\hline
\end{tabular}

Abbreviations: AN, acrylonitrile; APS, ammonium persulfate.

\section{Variation of monomer concentration}

The conversion and the rate of polymerization reaction increased with the increase in monomer concentration (0.5-3.5 mol dm $\left.\mathrm{dm}^{-3}\right)$, at fixed concentration of the other reagents and components. The results are presented in Table 1. From the double logarithmic plot of $R_{p} v s[A N]$, the $R_{p}$ was found to be 0.63 powers dependent on the monomer concentration. This order may be attributed to the greater rate of participation of the monomer in the initiation step. Less than one value of order has also been cited in the literature, as Chapiro ${ }^{11}$ found the monomer exponent varying from one to less than one, and the findings were attributed to the presence of impurity in the polymerization system. In addition, the deviation from unity or more than unity is normally observed as the polymerization system becomes heterogeneous in nature. ${ }^{12}$ The viscosity average molecular weight $(\mathrm{Mv})$ and the number average molecular weight $(\mathrm{Mn})$ of the polymer, as determined by viscosity and GPC methods, increased from $0.75 \times 10^{6}$ to $2.1 \times 10^{6}$, and $0.56 \times 10^{6}$ to $1.75 \times 10^{6}$ respectively, with increasing [AN] from 0.5 to $3.5 \times 10^{-2} \mathrm{~mol} \mathrm{dm}^{-3}$. 


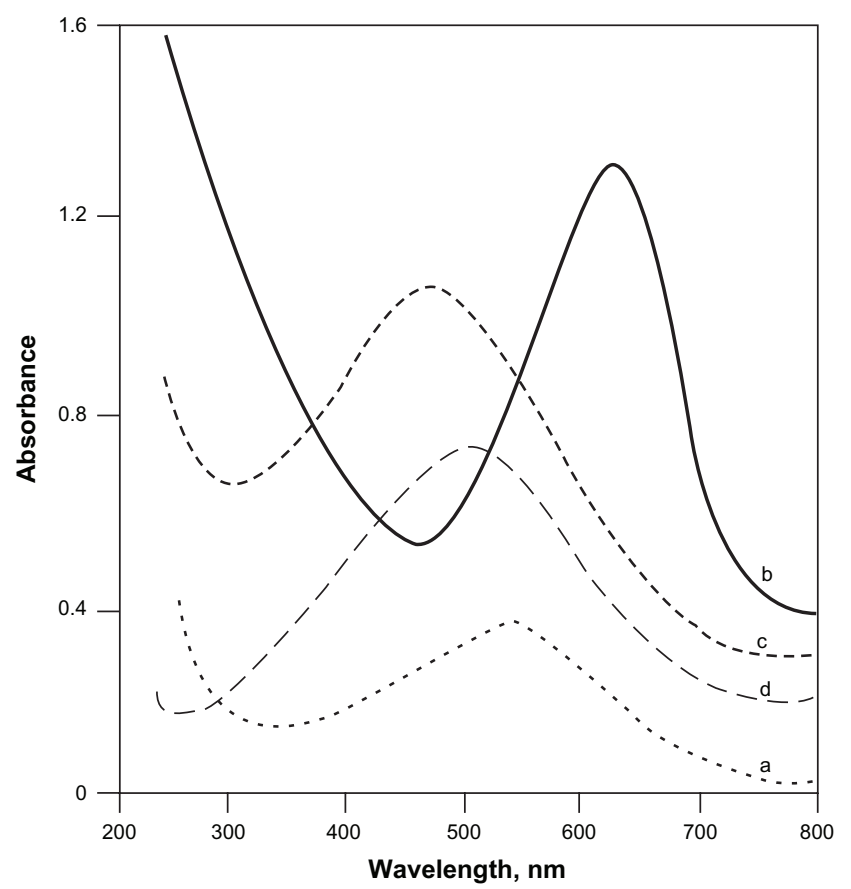

Figure I Spectral evidence for the interaction of Co-complex, APS, and AN a) $\mathrm{CoCl}_{2}$; b) $\left[\mathrm{Co}(\mathrm{III}) \mathrm{en}_{2} \mathrm{Cl}_{2}\right] \mathrm{Cl}$; c) $\left[\mathrm{Co}(\mathrm{III}) \mathrm{en}_{2} \mathrm{Cl}_{2}\right] \mathrm{Cl}+\mathrm{APS}+\mathrm{AN}$ before reaction; and d) $\left[\mathrm{Co}(\mathrm{III}) \mathrm{en}_{2} \mathrm{Cl}_{2}\right] \mathrm{Cl}+\mathrm{APS}+\mathrm{AN}$ after reaction.

Abbreviations: AN, acrylonitrile; APS, ammonium persulfate.

\section{Variation of initiator concentration}

The effects of the $R_{p}$ and the conversion have been studied by varying the concentration of the initiator in the range $2.5 \times 10^{-3}$ to $25.0 \times 10^{-3} \mathrm{~mol} \mathrm{dm}^{-3}$, as shown in Table 1 . With the increase in APS concentration in the above range, the conversion and $R_{p}$ were found to increase because of an

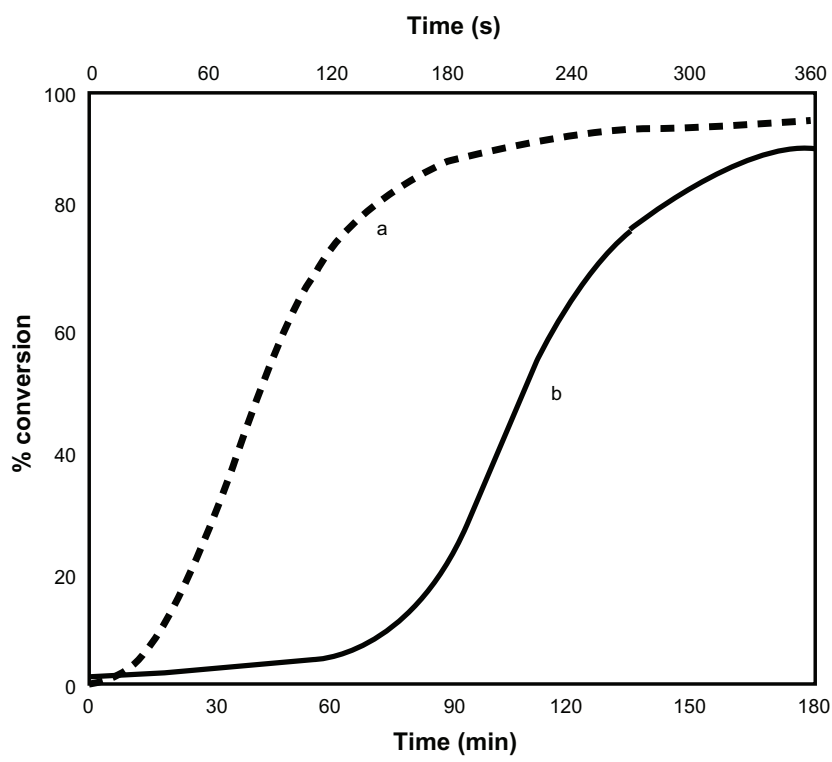

Figure $\mathbf{2} \%$ conversion PAN (a) in microwave oven and (b) in conventional heating. Abbreviation: PAN, polyacrylonitrile nanoparticles. increase in concentration of the active species resulting in smaller oligomers with higher critical micelle concentration. The double logarithmic plot gave a regular increasing trend with the order of 0.57 .

\section{Variation of $\left[\mathrm{Co}(\mathrm{III})(\mathrm{en})_{2} \mathrm{Cl}_{2}\right] \mathrm{Cl}$ concentration}

As Table 1 shows, it was found that the $\mathrm{R}_{\mathrm{p}}$ and \% conversion of $\mathrm{AN}$ were negligible in the absence of $\left[\mathrm{Co}(\mathrm{III})(\mathrm{en})_{2} \mathrm{Cl}_{2}\right] \mathrm{Cl}$ complex, both in conventional heating and in MW irradiation processes. However, the $\mathrm{R}_{\mathrm{p}}$ and \% conversion were found to increase uniformly with the increase in $\left[\mathrm{Co}(\mathrm{III}) \mathrm{en}_{2} \mathrm{Cl}_{2}\right] \mathrm{Cl}$ concentration (Table 1). The impact of $\left[\mathrm{Co}(\mathrm{III}) \mathrm{en}_{2} \mathrm{Cl}_{2}\right] \mathrm{Cl}$ on $\mathrm{R}_{\mathrm{p}}$ was found from the double logarithmic plot; the order of the reaction with respect to the $\left[\mathrm{Co}(\mathrm{III}) \mathrm{en}_{2} \mathrm{Cl}_{2}\right] \mathrm{Cl}$ concentration was observed to be 0.88 . The effect of the complex on $\mathrm{R}_{\mathrm{p}}$ has been described earlier. ${ }^{13}$ From the experimental data, it was observed that particle size increased with the increase in concentration of complex, as shown in Table 2. However, it is suggested that the polymerization was stabilized by the presence of the complex, with reduction of the particle size into the nano-range, which was further confirmed from TEM figure.

\section{Chain transfer}

Figure 3 shows the relationship between $\mathrm{R}_{\mathrm{p}}$ and the reciprocal of degree of polymerization (1/DP) of PAN observed when the [APS] was varied at a fixed [AN]. A linear relationship observed here excludes any significant chain transfer to the initiator in the polymerization of AN with APS and $\left[\mathrm{Co}(\mathrm{III}) \mathrm{en}_{2} \mathrm{Cl}_{2}\right] \mathrm{Cl}$.

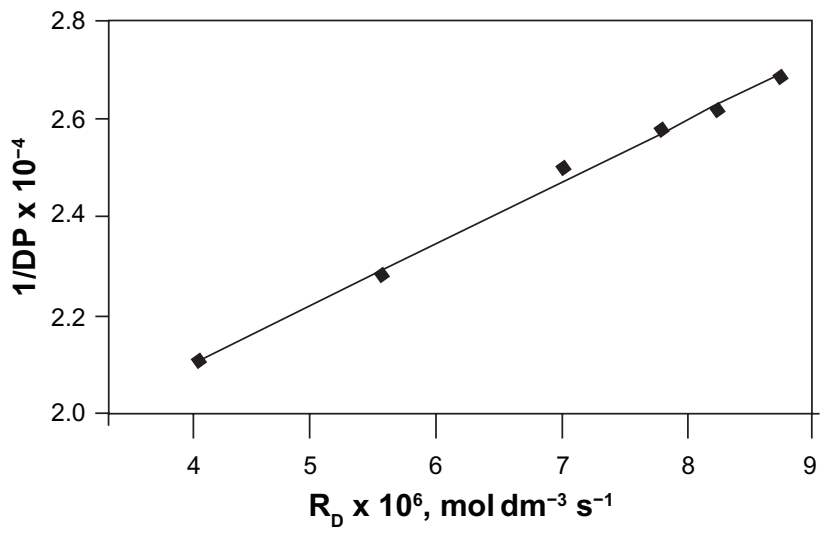

Figure 3 Dependence of the reciprocal of degree of polymerization (I/DP) of AN on Rp on varying [APS]; from $2.5 \times 10^{-3}$ to $25 \times 10^{-3} \mathrm{~mol} \mathrm{dm}^{-3}$ at $[A N]=2 \mathrm{~mol} \mathrm{dm}^{-3}$, and $\left[\mathrm{Co}(\mathrm{III}) \mathrm{en}_{2} \mathrm{Cl}_{2}\right] \mathrm{Cl}=10 \times 10^{-3} \mathrm{~mol} \mathrm{dm}^{-3}$.

Abbreviations: AN, acrylonitrile; APS, ammonium persulfate; Rp, rate of polymerization; I/DP, reciprocal of degree of polymerization. 


\section{Ultrasonic velocity}

Ultrasonic velocity data revealed that the sound velocity increases with the increase in molecular weight. From Figure 4, it is found that the sound velocity smoothly increases with increasing molecular weight of PAN without any more deviation, ie, the value is nearly uniform because of the uniform particle size. The data are in good agreement with the sound velocity data and a similar trend was noticed in the case of poly(ethylene glycol) as reported by Gerecze. ${ }^{14}$

\section{Mechanism}

The mode of initiator decomposition, chain initiation, and termination mechanism of polymerization involving the novel catalytic system $\left[\mathrm{Co}(\mathrm{III}) \mathrm{en}_{2} \mathrm{Cl}_{2}\right] \mathrm{Cl} / \mathrm{APS}$ can be interpreted as follows:

i. Production of free radicals $\left(\mathrm{SO}_{4}^{-\cdot}\right)$ from initiator decomposition catalyzed by $\mathrm{Co}(\mathrm{III})$ complex, as shown in Scheme 1;

ii. Production of free radicals in an aqueous phase to form oligomeric free radicals or to precipitate homogenously from the aqueous phase and to form colloidal particles;

iii. Capture of free radicals by monomer-swollen micelles or monomer droplets;

iv. Termination of free radicals in the aqueous phase, leading to capture efficiency less than $100 \%$.

The rate of complex catalysis has been found to be dependent on the proportions obtained as below, and the rate of expression is derived by applying the steady-state principle and assuming mutual termination:

$$
\mathrm{R}_{\mathrm{p}}=[\mathrm{AN}]^{0.63}[\mathrm{APS}]^{0.57}[\text { complex (I) }]^{0.88}
$$

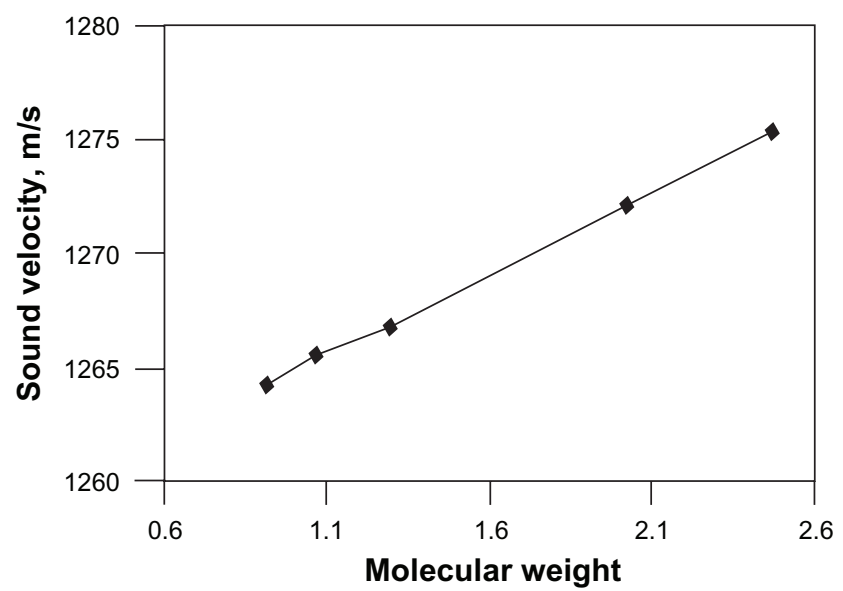

Figure 4 Variation of sound velocity with molecular weight of PAN solution in DMF at $30^{\circ} \mathrm{C}$.

Abbreviations: PAN, polyacrylonitrile nanoparticles; DMF, dimethylformamide.

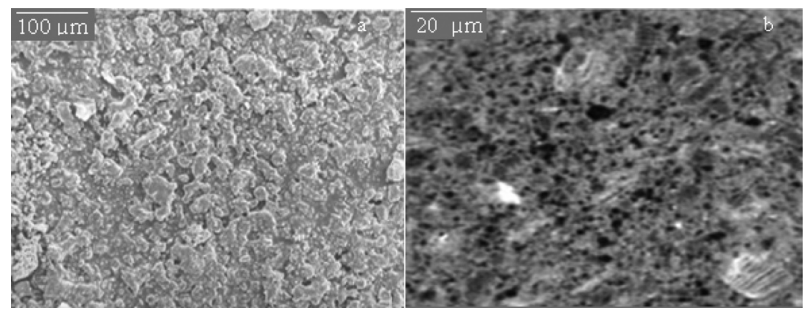

Figure 5 SEM of PAN nanoparticle prepared by MW irradiation at different magnification.

Abbreviations: SEM, scanning electron microscope; PAN, polyacrylonitrile nanoparticles; MW, microwave.

\section{SEM}

The characterization of PAN emulsion latex was performed by $\mathrm{SEM}$ of surface morphology. For the $\left[\mathrm{Co}(\mathrm{III}) \mathrm{en}_{2} \mathrm{Cl}_{2}\right] \mathrm{Cl} / \mathrm{AN} /$ APS $/ \mathrm{H}_{2} \mathrm{O}$ system, the SEM data for the particle size of the PAN latex is shown in Figure 5a and 5b at different magnifications. From Figure 5, it was found that the particle size was less than $100 \mathrm{~nm}$. Therefore, complex catalyzed polymerization can hopefully be utilized to prepare nano-scale latex particles. This was correlated with data provided in Table 2 and also further confirmed by TEM analysis as stated below.

\section{TEM}

The characterization of PAN latex was performed by TEM for particle size. The PAN nanoparticles were derived from TEM Figure 6. From this, it was found that the particle formed by microwave irradiation in the presence of complex was more uniform and smaller (Figure 6a) than the particle formed during thermal irradiation (Figure 6b), which was was due to the more uniform reaction. The results were similar to those reported by Bao and Zhang. ${ }^{15}$

\section{Conclusion}

The most significant outcome of the research described herein is the development of a novel, non-conventional initiating system leading to stabilizing of the emulsion latex

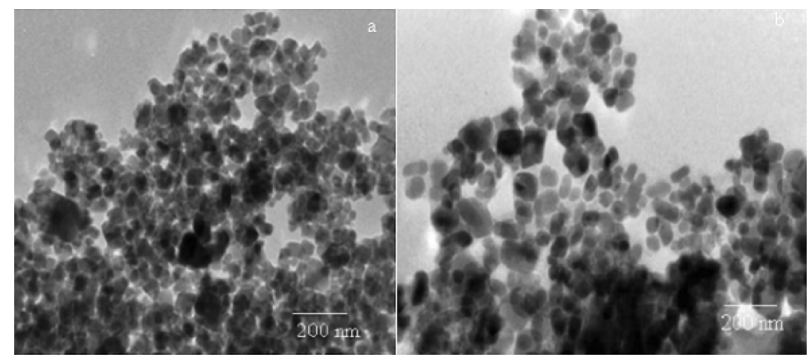

Figure 6 TEM of PAN nanoparticle by (a) MW irradiation and (b) conventional heating methods.

Abbreviations: TEM, transmission electron microscope; PAN, polyacrylonitrile nanoparticles; MW, microwave. 
to a high conversion in the presence of complex, through MW irradiation. The trans-[Co(III)en $\left.\mathrm{Cl}_{2}\right] \mathrm{Cl}$ complex most likely plays the role of surfactant in decreasing the latex particles to the nano-order scale. The polymer latex was characterized by TEM (size), SEM (morphology), and molecular weight measurements. Nano-scale latex particles could be obtained without emulsifier more easily than in a conventional emulsion polymerization. Hence, it is a promising technique for the preparation of the polymer nanoparticles/nanocomposites under investigation in our laboratory.

\section{Disclosure}

The authors report no conflicts of interest in this work.

\section{References}

1. Horvath IT, Anastas PT. Innovations and green chemistry. Chem Rev. 2007;107:2169-2173.

2. Fukuoka S, Tojo M, Hachiya H, Aminaka M, Hasegawa K. Green and sustainable chemistry in practice: development and industrialization of a novel process for polycarbonate production from $\mathrm{CO}_{2}$ without using phosgene. Polym J. 2007;39(2):91-114.

3. Galema SA. Microwave chemistry. Chem Soc Rev. 1997;26: 233-238.

4. Leadbeater NE, Torenius HM, Tye H. Microwave-promoted organic synthesis using ionic liquids: a mini review. Comb Chem High Throughput Screening. 2004;7:511-528.
5. Adachi K, Iwamura T, Chujo Y. Novel synthesis of submicrometer silica spheres in non-alcoholic solvent by microwave-assisted sol-gel method. Chem Lett. 2004;33(11):1504-1505.

6. Sahoo PK, Bhattacharya SP, Samal RK. Influence of $\mathrm{Cu}(\mathrm{II})$ salicylaldehyde couple on potassium monopersulphate decomposition and acrylonitrile polymerization in solution. Euro Polym J. 1985;21(5): 499-503.

7. Krishnamurthy M. Modified synthesis of trans-dichlorobisethylenediamine cobalt(III) chloride. J Inorg Nucl Chem. 1972;34: 3915-3916.

8. Sahoo PK, Mohapatra R. Synthesis and kinetic studies of PMMA nanoparticles by non-conventionally initiated emulsion polymerization. Euro Polym J. 2003;39:1839-1846.

9. Kurata M, Tsunashima Y. Polymer Handbook. 4th ed. Brandrup J, Immergut EH, editors. New York: Wiley; 1999.

10. Vogel AI. Vogel's Textbook of Quantitative Inorganic Analysis. 4th ed. Longman Science and Technology; 1980.

11. Chapiro A. Determination of free radical yield in the radiolysis of mixture by the polymerization method. J Chem Phys. 1995;63:801.

12. Odian G. Principles of Polymerization. 3rd ed. New York, NY: Wiley; 1981.

13. Sahoo PK, Samal B, Swain SK. Cobalt(III)-mediated microemulsion polymerization of acrylonitrile: Kinetics and particle morphology. J Appl Polym Sci. 2004;91:3120-3126.

14. Gerecze NG. Ultrasonic studies in solution of poly(ethylene glycol) Acoustica. 1977;38:51-57.

15. Bao J, Zhang A. Poly(methyl methacrylate) manoparticles prepared through microwave emulsion polymerization. J Appl Polym Sci. 2004;93:2815-2820.
Nanotechnology, Science and Applications

\section{Publish your work in this journal}

Nanotechnology, Science and Applications is an international, peerreviewed, open access journal that focuses on the science of nanotechnology in a wide range of industrial and academic applications. It is characterized by the rapid reporting across all sectors, including engineering, optics, bio-medicine, cosmetics, textiles, resource sustainability

\section{Dovepress}

and science. Applied research into nano-materials, particles, nanostructures and fabrication, diagnostics and analytics, drug delivery and toxicology constitute the primary direction of the journal. The manuscript management system is completely online and includes a very quick and fair peer-review system, which is all easy to use. 